\title{
SUSY searches with taus at the LHC
}

\author{
Feng Lyu ${ }^{1 \star}$ on behalf of ATLAS and CMS Collaborations
}

1 Institute of High Energy Physics, Chinese Academy of Sciences

$\star$ luf@ihep.ac.cn

Proceedings for the 15th International Workshop on Tau Lepton Physics, Amsterdam, The Netherlands, 24-28 September 2018

doi:10.21468/SciPostPhysProc. 1

\begin{abstract}
The supersymmetric partner of the tau lepton, the stau, is predicted to be relatively light in a range of SUSY models and may be a key for dark matter. This talk presents recent ATLAS and CMS results from proton-proton collisions at $\sqrt{s}=13 \mathrm{TeV}$ corresponding to an integrated luminosity of $36.1 \mathrm{fb}^{-1}$ and $35.9 \mathrm{fb}^{-1}$ delivered by the LHC and recorded by the ATLAS and CMS detectors respectively in 2015 and 2016, to search for direct stau pair production, and indirect stau production mediated by other SUSY particles.
\end{abstract}

Copyright F. Lyu et al.

This work is licensed under the Creative Commons

Attribution 4.0 International License.

Published by the SciPost Foundation.
Received 13-11-2018

Accepted 17-01-2019

Published 20-02-2019

doi:10.21468/SciPostPhysProc.1.023

\section{Contents}

1 Introduction $\quad 2$

2 SUSY strong production processes $\quad 2$

2.1 The first and second squark or gluino pairs to taus 2

2.2 Stop pair to taus 4

3 SUSY electroweak production processes 4

$\begin{array}{lll}3.1 & \text { Direct/indirect staus } & 4\end{array}$

$\begin{array}{lll}3.2 & \text { Indirect stau to taus } & 6\end{array}$

$\begin{array}{lll}3.3 \text { Gauginos to } 3 / 4 \mathrm{~L} \text { ( up to } 2 \tau_{h} \text { ) } & 7\end{array}$

3.4 Gauginos RPV and RPC 4L ( up to $2 \tau_{h}$ ) 8

4 Conclusion $\quad 10$

$\begin{array}{ll}\text { References } & 11\end{array}$ 


\section{Introduction}

The Standard Model (SM) of particle physics is very successful so far, however it can only cover about $5 \%$ of the universe, and still many phenomena and theoretical problems are unexplained, such as: Dark matter and dark energy; matter/anti-matter asymmetry; neutrino masses/mixing; hierarchy problem; gravity in gauge theory and its unification, and so on. The SUSY theoretical hypothesis, based on a unique symmetry which relates matter and forces particles (fermions and bosons), can solve most of the current puzzles. SUSY could be a powerful key to unification and cosmology, the discovery of its 'hidden world' can bring a great revolution of modern physics in the 21 st century.

Final states with taus are of particular interest in SUSY searches: firstly, $\tilde{\tau}$ is a superpartner of the third generation fermion $\tau$, it's a colorless scalar, which is predicted to be light in SUSY scenarios and leads to $\tau$-riched final states; secondly, models with light staus can lead to a darkmatter relic density consistent with cosmological observations; finally, independent studies of $\tau$ s channels are necessary to investigate the coupling structure of the new physics, potentially discovered in leptonic final states, especially with regard to lepton universality.

SUSY particles can be created by strong and electroweak processes at the Large Hadron Collider (LHC) [1]. All analyses results in this talk come from proton-proton collisions at $\sqrt{s}$ $=13 \mathrm{TeV}$ corresponding to an integrated luminosity of $36.1 \mathrm{fb}^{-1}$ and $35.9 \mathrm{fb}^{-1}$ delivered by the LHC and recorded by the ATLAS [2] and CMS [3] detector respectively in 2015 and 2016. SM processes are backgrounds for SUSY searches, we need firstly to carefully understand and accurately model the SM backgrounds, then to determine SUSY signals' existence by observing significant events excess above SM level in LHC real data. So far, all results in this talk don't observe such exciting excess in their signal regions after well understanding SM backgrounds by various control regions and validation regions. Therefore limits at the $95 \%$ confidence level (CL) on the model parameters are set.

\section{SUSY strong production processes}

\subsection{The first and second squark or gluino pairs to taus}

An inclusive search for squarks and gluinos produced via the strong interaction in events with jets, at least one hadronically decaying to $\tau$-lepton, and large missing transverse momentum is performed by the ATLAS Collaboration [4]. Two SUSY models are considered: a simplified model [5-7] of gluino pair production and a model of gauge-mediated SUSY breaking (GMSB) [8-10], see Figure 1. One $\tau$-lepton $(1 \tau)$ or at least two $\tau$-leptons $(2 \tau)$ provide complementary acceptance to SUSY signals. The search strategies for these two channels are optimized separately and the results are statistically combined.

The observed data are consistent with background expectations from the Standard Model. Upper limits are set at 95\% confidence level on the number of events that could be produced by processes beyond the Standard Model. Results are also interpreted in the framework of a simplified model of gluino pairs decaying into $\tau$-leptons via $\tau$-sleptons, and a minimal model of gauge-mediated supersymmetry breaking with the lighter $\tau$-slepton as the Next-to-LightestSupersymmetric-Particle (NLSP) at large $\tan \beta$. At 95\% CL in the simplified model, gluino masses up to $2000 \mathrm{GeV}$ are excluded for low LSP masses, and LSP masses up to $1000 \mathrm{GeV}$ are excluded for gluino masses around $1400 \mathrm{GeV}$ (see Figure 2). In the GMSB model, values of the SUSY-breaking scale $\Lambda$ below $110 \mathrm{TeV}$ are excluded at 95\% CL for all values of $\tan \beta$ in the range $2 \leq \tan \beta \leq 60$, while a stronger limit of $120 \mathrm{TeV}$ is achieved for $\tan \beta>30$ (see Figure 3). 

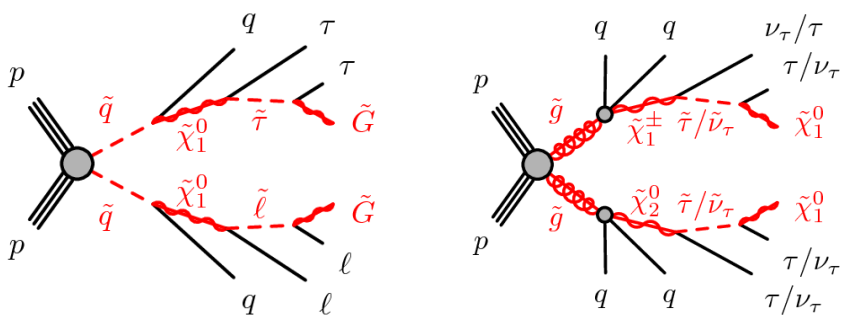

Figure 1: Diagrams illustrating example processes of the GMSB model (left), the scalar lepton $\tilde{l}$ is preferentially a $\tilde{\tau}_{1}$ for high values of $\tan \beta$, and the simplified model (right) [4].

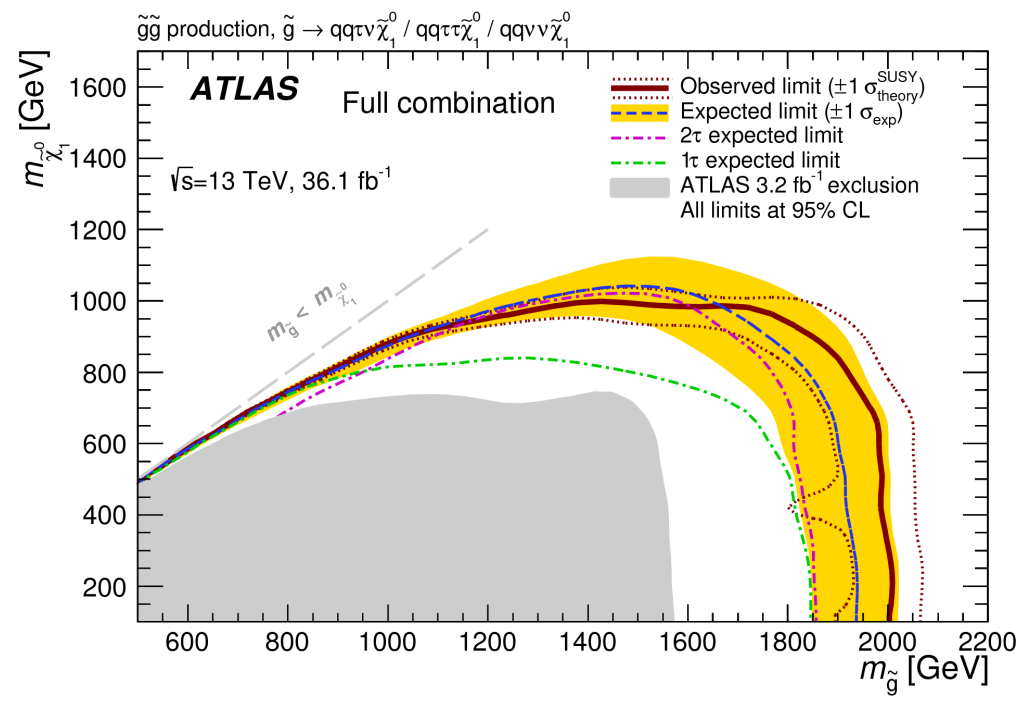

Figure 2: Exclusion contours at the 95\% CL as a function of the LSP mass $m_{\tilde{\chi}_{1}^{0}}$ and gluino mass $m_{\tilde{g}}$ for the simplified model of gluino pair production [4].

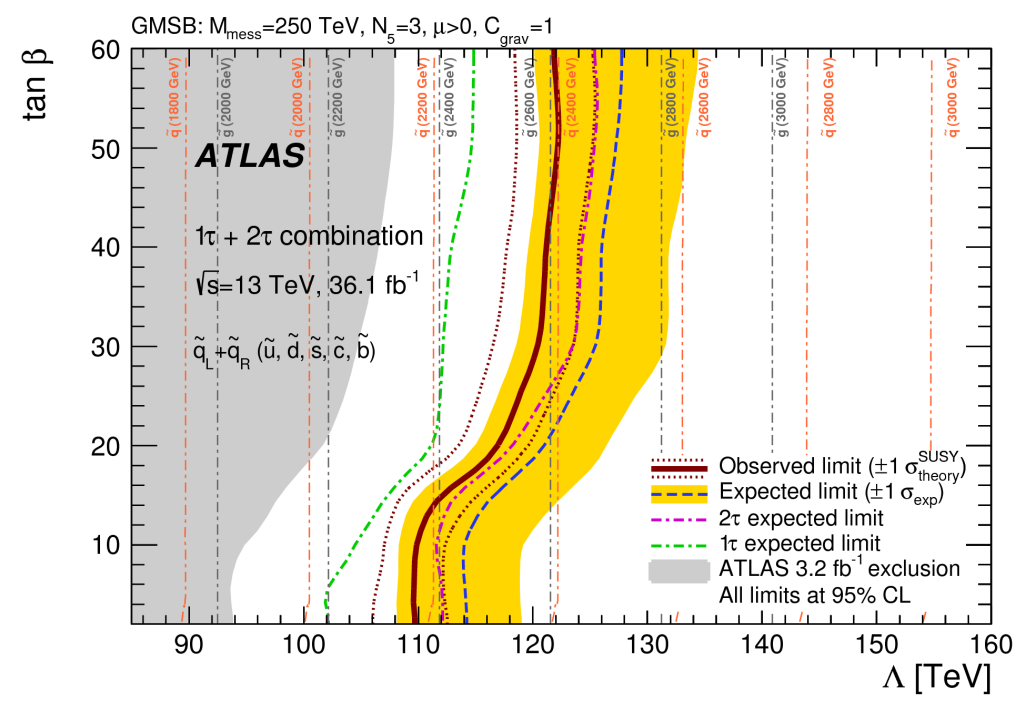

Figure 3: Exclusion contours at the $95 \%$ CL as a function of $\tan \beta$ and the SUSYbreaking mass scale $\Lambda$ for the gauge-med- iated supersymmetry-breaking model [4]. 


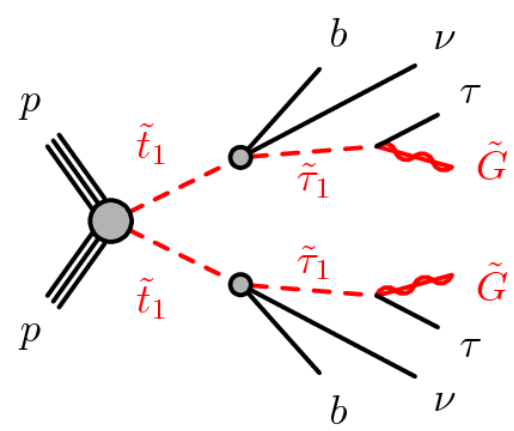

Figure 4: The simplified model for production and decay of SUSY particles considered [11].

\subsection{Stop pair to taus}

The ATLAS analysis in Ref. [11] describes a search for SUSY in a benchmark scenario motivated by gauge-mediated SUSY breaking [8-10] and natural gauge mediation [12]. In this scenario, only three sparticles are assumed to be sufficiently light to be relevant for the phenomenology at LHC: the lightest scalar partner of the top quark (top squark, $\tilde{t}_{1}$ ), the lightest scalar partner of the tau lepton (tau slepton, $\tilde{\tau}_{1}$ ), and a nearly massless gravitino $\tilde{G}$. The search strategy is optimized using a simplified model $[6,7]$ with this limited sparticle content. The relevant parameters are the sfermion masses $m\left(\tilde{t}_{1}\right)$ and $m\left(\tilde{\tau}_{1}\right)$. The process is illustrated in Figure 4. The top squark is assumed to be light $[13,14]$ and directly pair-produced through the strong interaction. Each top squark decays to a b-quark, a tau neutrino, and a tau slepton which in turn decays to a tau lepton and a gravitino. The branching ratios for these decays are set to $100 \%$, and the decays are assumed to be prompt. The tau-slepton mixing matrix is chosen such that the tau slepton is an equal mix of the superpartners of the left- and the right-handed tau lepton.

In this ATLAS analysis, a search is presented for the direct pair production of supersymmetric top squarks in final states with two tau leptons, jets identified as originating from b-hadron decays, and missing transverse momentum. Two exclusive channels are considered, which select events with either two hadronically decaying tau leptons or one hadronically decaying tau lepton and one electron or muon. Good agreement between the Standard Model prediction and the event yield observed in data is found in the signal region of each channel. The analysis results are therefore interpreted in terms of upper limits on the production of supersymmetric particles. In a simplified model with production of two top squarks, each decaying via a tau slepton to a nearly massless gravitino as the lightest supersymmetric particle, masses up to $\mathrm{m}\left(\tilde{t}_{1}\right)=1.16 \mathrm{TeV}$ and $\mathrm{m}\left(\tilde{\tau}_{1}\right)=1.00 \mathrm{TeV}$ are excluded at $95 \%$ confidence level (see Figure 5). This result represents an improvement of the previous limits by almost a factor of two. Model-independent limits allow the exclusion of visible cross sections above $0.15(0.13) \mathrm{fb}$ in the leponic-hadronic (fully hadronic) channel.

\section{SUSY electroweak production processes}

\subsection{Direct/indirect staus}

This CMS analysis [15] examines simplified SUSY models in which the $\tilde{\tau}$ can be produced either directly, through pair production, or indirectly, in the decay chains of charginos and neutralinos. In all cases, we assume that the $\tilde{\tau}$ decays to a $\tau$ lepton and $\tilde{\chi}_{1}^{0}$. Diagrams illustrating these simplified models of direct and indirect $\tilde{\tau}$ production are shown in Figure 6. 


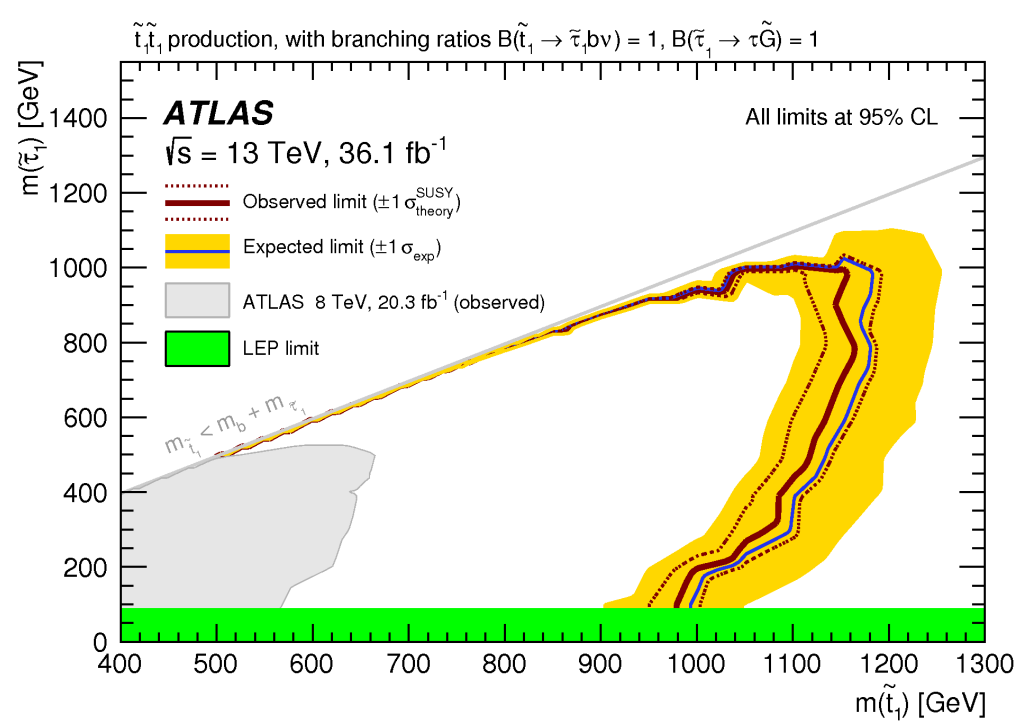

Figure 5: Exclusion contours at 95\% CL in the plane of top-squark and tau-slepton mass for the simplified model [11].
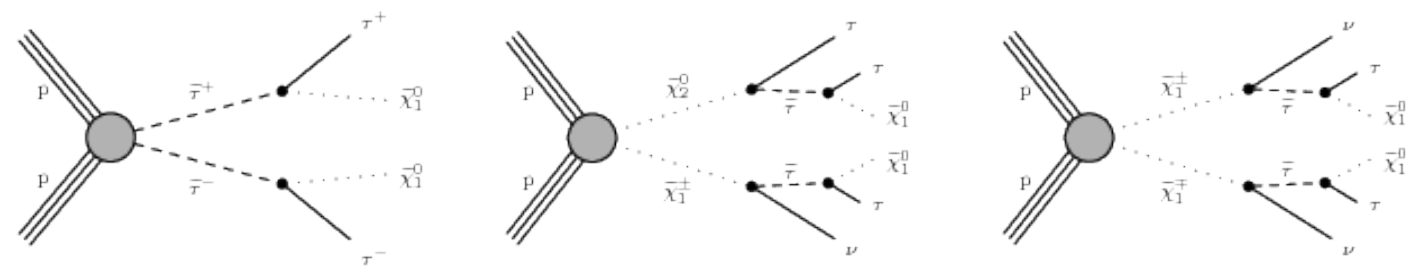

Figure 6: Diagrams for the simplified models: direct $\tilde{\tau}$ pair production followed by each $\tilde{\tau}$ decaying to a $\tau$ lepton and $\tilde{\chi}_{1}^{0}$ (left), and chargino-neutralino (middle) and chargino pair (right) production with subsequent decays leading to $\tau$ leptons in the final state [15].

This CMS search for the direct and indirect production of $\tau$ sleptons has been performed with a $\tau$ lepton pair and significant missing transverse momentum in the final state. Both leptonic and hadronic decay modes of the $\tau$ leptons are considered. Search regions are defined using discriminating kinematic observables that exploit expected differences between signal and background. No excess above the expected SM background has been observed. Upper limits on the cross section of direct $\tilde{\tau}$ pair production are derived for simplified models in which each $\tilde{\tau}$ decays to a $\tau$ lepton and the lightest neutralino, with the latter assumed to be the lightest supersymmetric particle (LSP). The analysis is most sensitive to a $\tilde{\tau}$ that is purely left-handed. For a left-handed $\tilde{\tau}$ of $90 \mathrm{GeV}$ decaying to a nearly massless LSP, the observed limit is 1.26 times the expected production cross section in the simplified model. The limits obtained for direct $\tilde{\tau}$ pair production represent a considerable improvement in sensitivity for this production mechanism with respect to previous LHC measurements. Exclusion limits are also derived for simplified models of chargino-neutralino and chargino pair production with decays to $\tau$ leptons that involve indirect $\tilde{\tau}$ production via the chargino and neutralino decay chains. In the chargino-neutralino production model, in which the parent chargino and second-lightest neutralino are assumed to have the same mass, chargino masses up to $710 \mathrm{GeV}$ are excluded under the hypothesis of a nearly massless LSP (see Figure 7). In the chargino pair production model, chargino masses up to $630 \mathrm{GeV}$ are excluded under the same hypothesis (see Figure 8). 


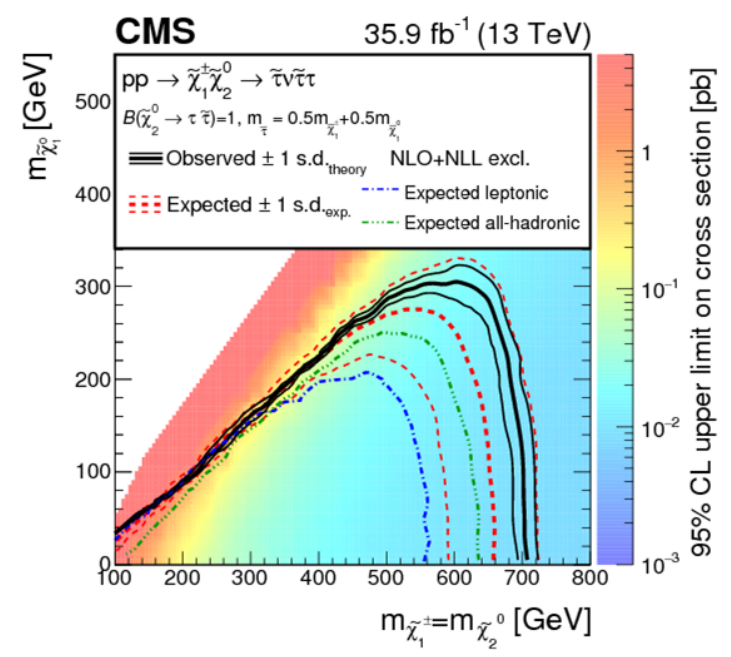

Figure 7: Exclusion limits at 95\% CL for chargino-neutralino production with decays through $\tilde{\tau}$ to final states with $\tau$ leptons [15].

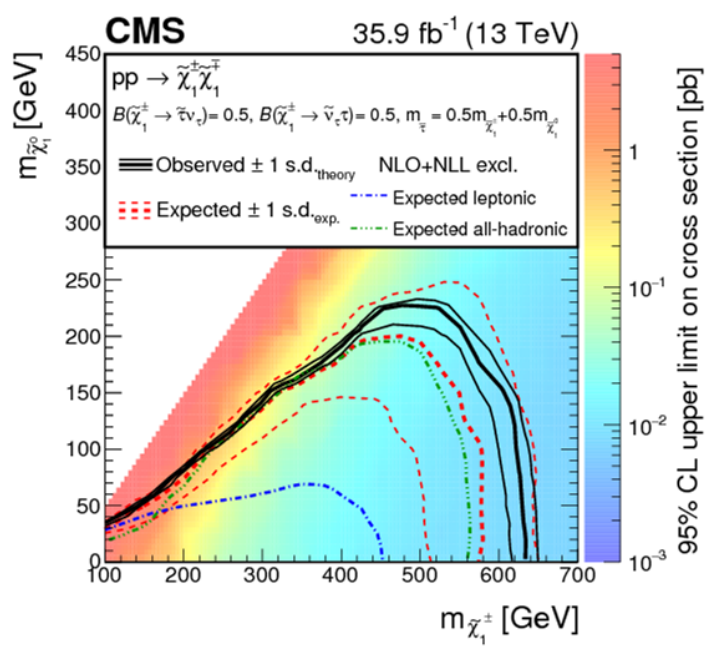

Figure 8: Exclusion limits at 95\% CL for chargino pair production with decays through $\tilde{\tau}$ to final states with $\tau$ leptons [15].

\subsection{Indirect stau to taus}

This ATLAS analysis [16] considers scenarios where the production of charginos, neutralinos, and sleptons may dominate at the LHC with respect to the production of squarks and gluinos which can be realised in the general framework of the phenomenological Minimal Supersymmetric Standard Model (pMSSM) [17,18]. Two simplified models $[5,6,19]$ of $\tilde{\chi}_{1}^{+} \tilde{\chi}_{1}^{-}$and $\tilde{\chi}_{1}^{ \pm} \tilde{\chi}_{2}^{0}$ production are considered in this work. The models are designed to enhance the probability of experimental observation. In both models, the lightest neutralino is the LSP and purely bino, the stau and tau sneutrino are assumed to be mass-degenerate, and the $\tilde{\tau}_{1}$ is assumed to be purely left-handed stau $\left(\tilde{\tau}_{L}\right)$. The mass of the $\tilde{\tau}_{L}$ state is set to be halfway between the masses of the $\tilde{\chi}_{1}^{ \pm}$and $\tilde{\chi}_{1}^{0}$, i.e. $m\left(\tilde{\tau}_{L}\right)=m\left(\tilde{\chi}_{1}^{0}\right)+\mathrm{x} \cdot\left(m\left(\tilde{\chi}_{1}^{ \pm}\right)-m\left(\tilde{\chi}_{1}^{0}\right)\right)$, with the parameter $\mathrm{x}=0.5$. Other values of $\mathrm{x}$ are also studied for selected benchmark models where $\mathrm{x}$ is varied between 0.05 and 0.95 in steps of 0.1 . All sparticles other than those explicitely mentioned here are assumed to be inaccessible at the LHC energy. In the model characterised by $\tilde{\chi}_{1}^{ \pm} \tilde{\chi}_{2}^{0}$ production, the $\tilde{\chi}_{1}^{ \pm}$ and $\tilde{\chi}_{2}^{0}$ are assumed to be pure wino and mass-degenerate. In the model where only $\tilde{\chi}_{1}^{+} \tilde{\chi}_{1}^{-}$ production is considered, the $\tilde{\chi}_{1}^{ \pm}$is pure wino. The above assumptions guarantee large pro- 

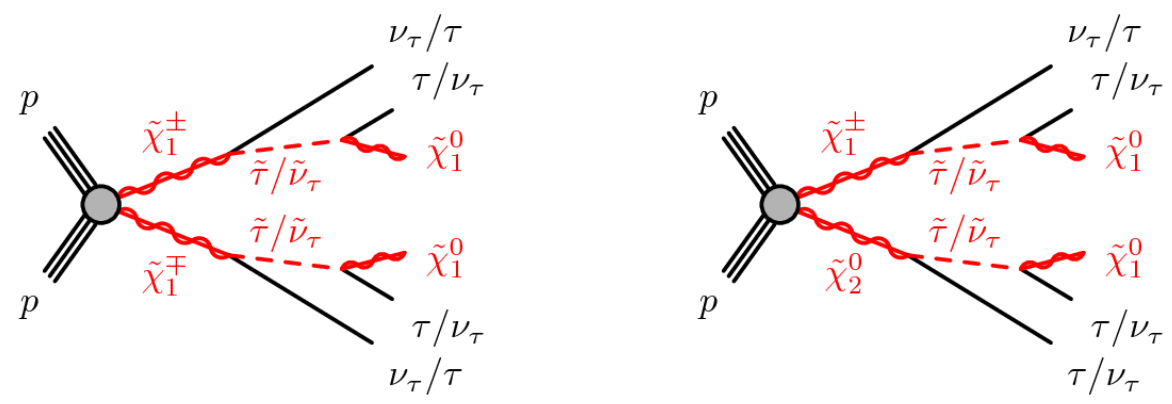

Figure 9: Representative diagrams for the electroweak production and decay processes of supersymmetric particles considered: (left) $\tilde{\chi}_{1}^{+} \tilde{\chi}_{1}^{-}$and (right) $\tilde{\chi}_{1}^{ \pm} \tilde{\chi}_{2}^{0}$ production [16].
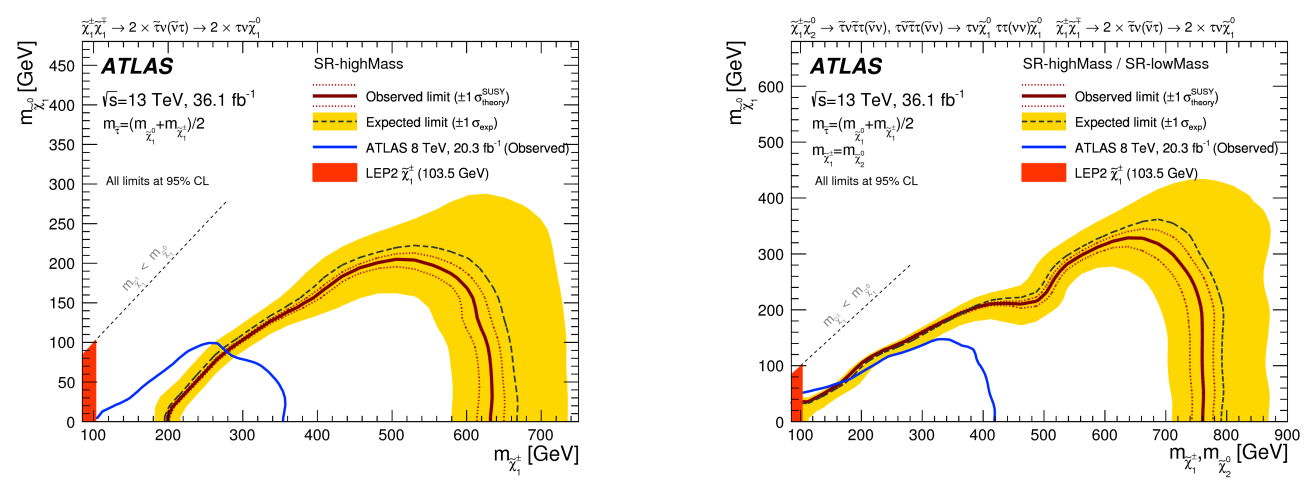

Figure 10: The 95\% CL exclusion contours for simplified models with $\tilde{\chi}_{1}^{+} \tilde{\chi}_{1}^{-}$production (left) and production of $\tilde{\chi}_{1}^{+} \tilde{\chi}_{1}^{-}$and $\tilde{\chi}_{1}^{ \pm} \tilde{\chi}_{2}^{0}$ (right) [16].

duction cross sections and short decay chains for $\tilde{\chi}_{1}^{ \pm}$and $\tilde{\chi}_{2}^{0}$. Charginos and next-tolightest neutralinos decay into the lightest neutralino via an intermediate on-shell stau or tau sneutrino, $\tilde{\chi}_{1}^{ \pm} \longrightarrow \tilde{\tau} v_{\tau}\left(\tilde{v}_{\tau} \tau\right) \longrightarrow \tau v_{\tau}\left(v_{\tau} \tau\right) \tilde{\chi}_{1}^{0}, \tilde{\chi}_{2}^{0} \longrightarrow \tilde{\tau} \tau \longrightarrow \tau \tau \tilde{\chi}_{1}^{0}$, and $\tilde{\chi}_{2}^{0} \longrightarrow \tilde{v}_{\tau} v_{\tau} \longrightarrow v_{\tau} v_{\tau} \tilde{\chi}_{1}^{0}$ (see Figure 9).

Agreement between data and SM predictions is observed in two optimised signal regions. Exclusion limits are placed on parameters of simplified electroweak supersymmetry models in scenarios where the neutralinos and charginos decay solely via intermediate left-handed staus and tau sneutrinos, and the mass of the $\tilde{\tau}_{L}$ state is set to be halfway between the masses of the $\tilde{\chi}_{1}^{ \pm}$and the $\tilde{\chi}_{1}^{0}(\mathrm{x}=0.5)$. Chargino masses up to $630 \mathrm{GeV}$ are excluded for a massless lightest neutralino in the scenario of direct production of chargino pairs, with each chargino decaying into the lightest neutralino via an intermediate on-shell stau or tau sneutrino (see Figure 10 left). An additional benchmark scenario with large mass-splitting $m\left(\tilde{\chi}_{1}^{ \pm}\right)=600 \mathrm{GeV}$ and massless $\tilde{\chi}_{1}^{0}$ ) can be excluded for $\mathrm{x}$ up to 0.75 , whereas a compressed benchmark scenario $m\left(\tilde{\chi}_{1}^{ \pm}\right)=250 \mathrm{GeV}$ and $\left.m\left(\tilde{\chi}_{1}^{0}\right)=100 \mathrm{GeV}\right)$ can only be excluded for the extreme cases with $\mathrm{x}=$ 0.05 or $\mathrm{x}=0.95$. In the case of production of chargino pairs and mass-degenerate charginos and next-to-lightest neutralinos, common $\tilde{\chi}_{1}^{ \pm}$and $\tilde{\chi}_{1}^{0}$ masses up to $760 \mathrm{GeV}$ are excluded for a massless lightest neutralino (see Figure 10 right). The additional benchmark scenarios with small and large mass-splitting can be both excluded for all considered values of $\mathrm{x}$.

\subsection{Gauginos to $3 / 4 \mathrm{~L}$ ( up to $2 \tau_{h}$ )}

This CMS analysis, described in Ref. [20], performed a search for direct production of charginos and neutralinos, mixtures of the SUSY partners of the electroweak gauge and Higgs bosons, decaying to two, three, or more charged leptons, and significant missing transverse momentum 

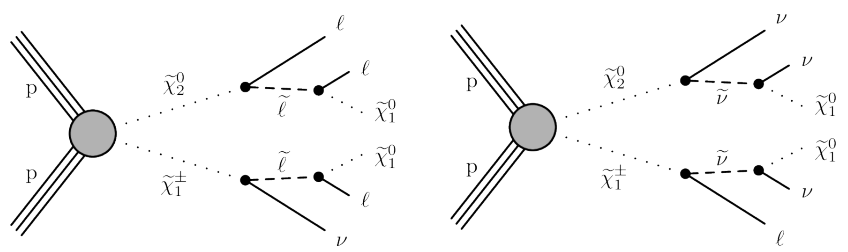

Figure 11: Chargino and neutralino pair production with decays mediated by sleptons and sneutrinos [20].
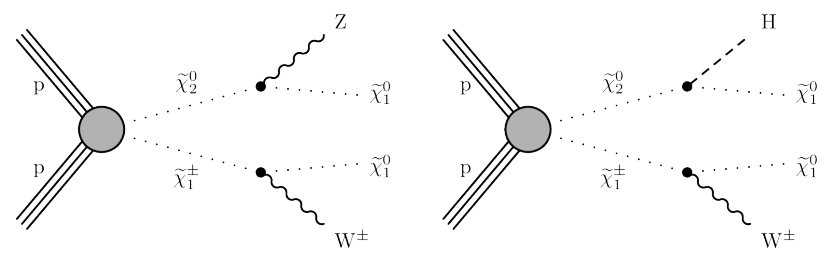

Figure 12: Chargino and neutralino pair production with the chargino decaying to a $\mathrm{W}$ boson and the LSP and the neutralino decaying to (left) a Z boson and the LSP or (right) a Higgs boson and the LSP [20].
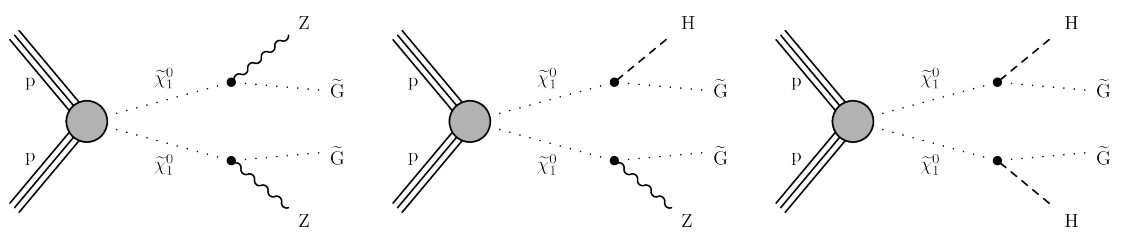

Figure 13: A GMSB model with higgsino pair production. The $\tilde{\chi}_{2}^{0}, \tilde{\chi}_{1}^{ \pm}$and $\tilde{\chi}_{1}^{0}$ are nearly mass degenerate with $\tilde{\chi}_{1}^{0}$ decaying to Z or Higgs bosons and $\tilde{G}$ LSP [20].

$\left(p_{T}^{\text {miss }}\right)$. In events with two light leptons (electrons or muons), the leptons are required to have the same charge; in events with three or more leptons, up to two may be hadronically decaying tau leptons $\left(\tau_{h}\right)$. Related diagrams are show in Figures 11, 12 and 13. The observed event yields are consistent with the expectations based on the standard model. The results are interpreted in simplified models of supersymmetry describing various scenarios for the production and decay of charginos and neutralinos. Depending on the model parameters chosen, mass values between $180 \mathrm{GeV}$ and $1150 \mathrm{GeV}$ are excluded at 95\% CL (see Figures 14, 15 and 16).

\subsection{Gauginos RPV and RPC 4L ( up to $2 \tau_{h}$ )}

This ATLAS analysis in Ref. [21] addresses a scenario with concurrent R-parity conserving (RPC) and violating (RPV) processes. Diagrams of the benchmark SUSY models of RPC NLSP pair-production in Figures 17, 18, 19 and 20. They involve wino (Figures 17 and 18), sleptonsneutrino (Figure 19) or gluino (Figure 20) production followed by the RPV decay of the $\tilde{\chi}_{1}^{0}$ LSP. The LSP is assumed to decay as $\tilde{\chi}_{1}^{0} \longrightarrow l l v$ with $100 \%$ branching ratio. The diagrams of the processes in the SUSY RPC General Gauge Mediated (GGM) higgsino model, the W*/Z* produced in the $\tilde{\chi}_{1}^{ \pm} / \tilde{\chi}_{2}^{0}$ decays are off-shell $(m \sim 1 \mathrm{GeV})$ and their decay products are usually not reconstructed (see Figures 21 and 22).

Four-lepton signal regions with up to two hadronically decaying taus are designed to target a range of supersymmetric scenarios that can be either enriched or depleted in events involving the production and decay of a Z boson. Data yields are consistent with SM expectations and results are used to set upper limits on the event yields from processes beyond the Standard Model. Exclusion limits are set at the 95\% confidence level in simplified models of General 


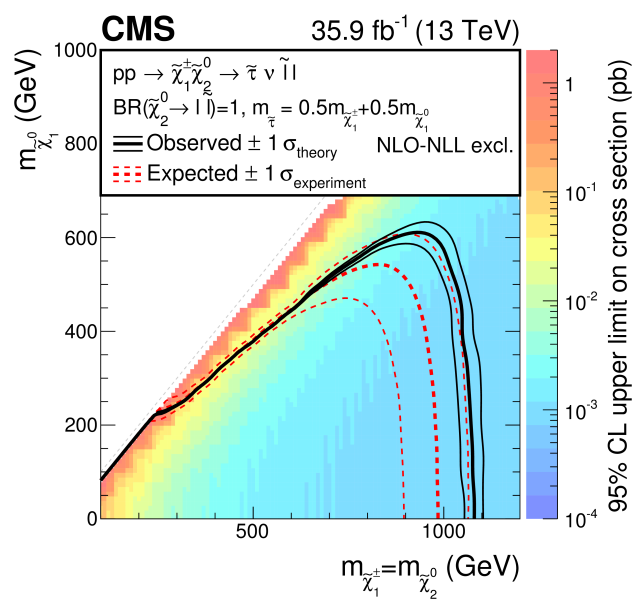

Figure 14: Interpretation of the results in the $\tau$-enriched model with mass parameter $\mathrm{x}=0.5[20]$.

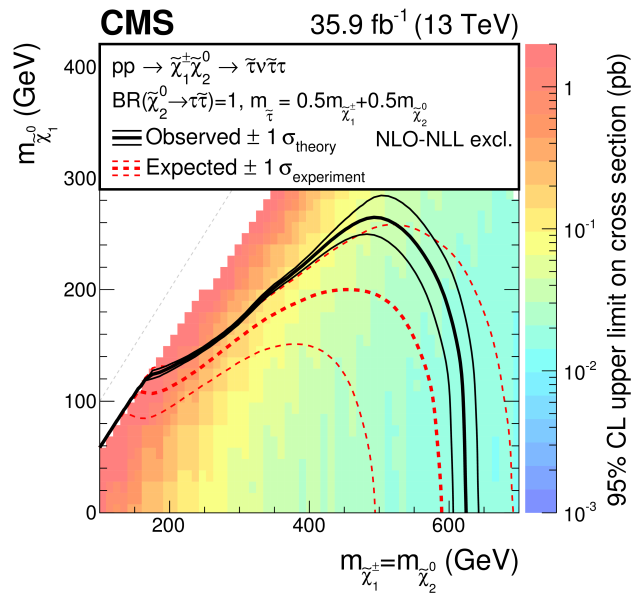

Figure 15: Interpretation of the results in the $\tau$-dominated model with mass parameter $\mathrm{x}=0.5[20]$.

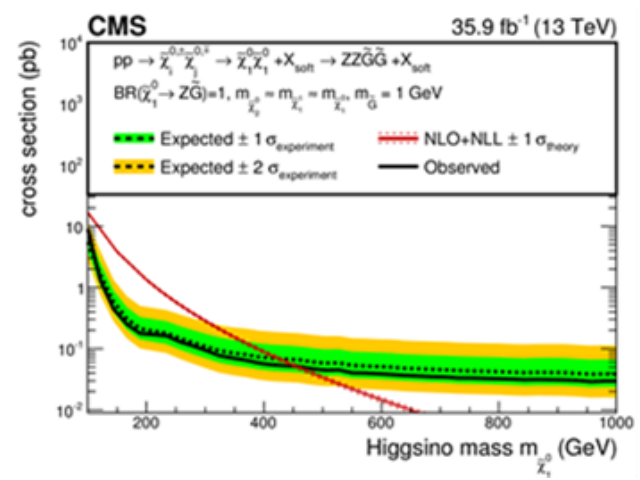

Figure 16: Interpretation of the results in the $\tilde{\chi}_{1}^{0} \tilde{\chi}_{1}^{0} \longrightarrow \mathrm{ZZ} \tilde{G} \tilde{G}$ model [20].

GGM SUSY, where higgsino masses are excluded up to $295 \mathrm{GeV}$. In R-parity-violating simplified models with decays of the lightest supersymmetric particle to charged leptons, lower limits of $1.46 \mathrm{TeV}, 1.06 \mathrm{TeV}$, and $2.25 \mathrm{TeV}$ are placed on wino, slepton and gluino masses, respectively (see Figures 23, 24, 25, 26 and 27). 


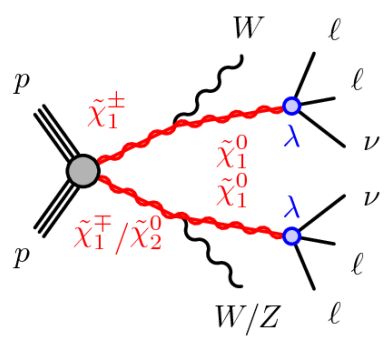

Figure 17: Wino W/Z NLSP

[21].

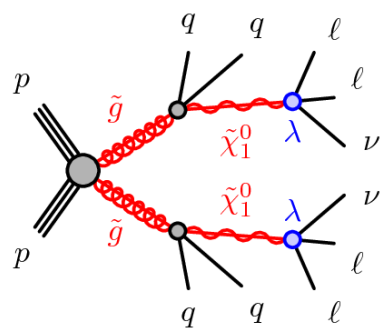

Figure 20: $\tilde{g}$ NLSP [21].

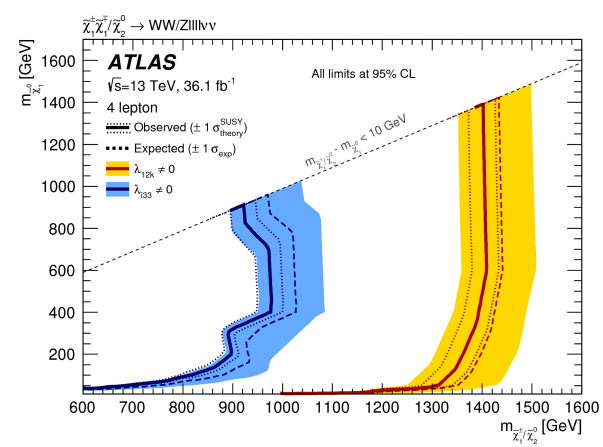

Figure 23: RPV wino W/Z NLSP [21].

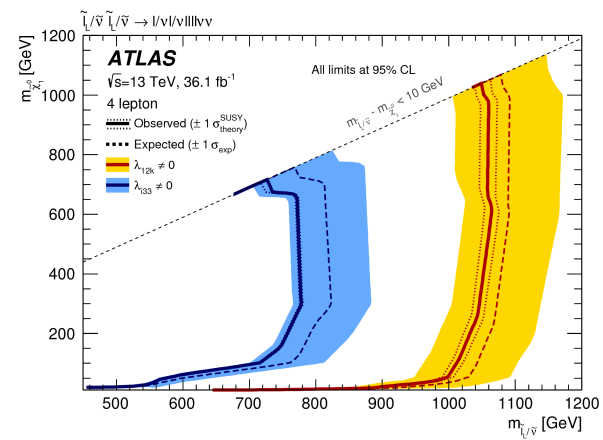

Figure 25: RPV $\tilde{l} / \tilde{v}$ NLSP [21].

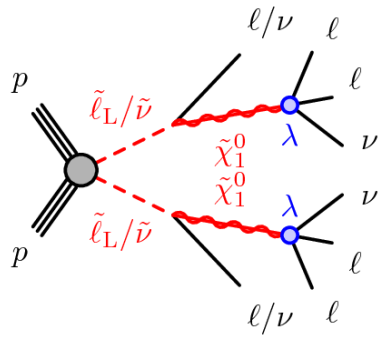

Figure 19: $\tilde{l}_{L} / \tilde{v}$ NLSP

[21].

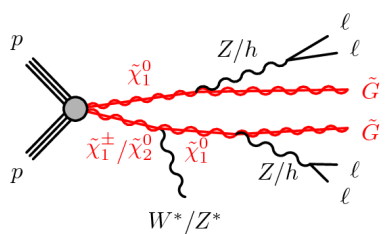

Figure 22: $\tilde{\chi}_{1}^{0} \tilde{\chi}_{1}^{ \pm} / \tilde{\chi}_{2}^{0}[21]$.

Figure 21: $\tilde{\chi}_{1}^{\mp} \tilde{\chi}_{1}^{ \pm} / \tilde{\chi}_{2}^{0}[21]$.

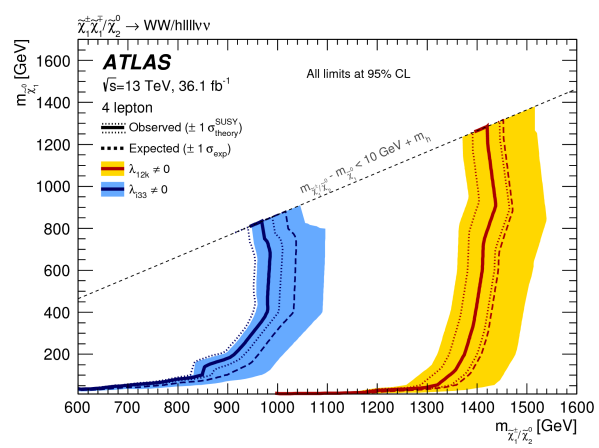

Figure 24: RPV wino W/h NLSP [21].

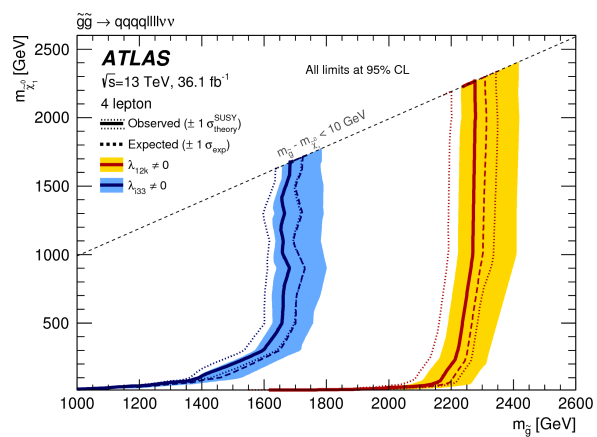

Figure 26: RPV $\tilde{g}$ NLSP [21].

\section{Conclusion}

The above six results from ATLAS and CMS for SUSY searches with taus at the LHC under $\sqrt{s}$ $=13 \mathrm{TeV}$ by an integrated luminosity of $36.1 \mathrm{fb}^{-1}$ and $35.9 \mathrm{fb}^{-1}$ respectively are included, no significant deviation from SM is observed, so 95\% CL limits are set. Model-independent upper limits on the SUSY-tau cross section are set; SUSY masses exclusion limits are set for the 


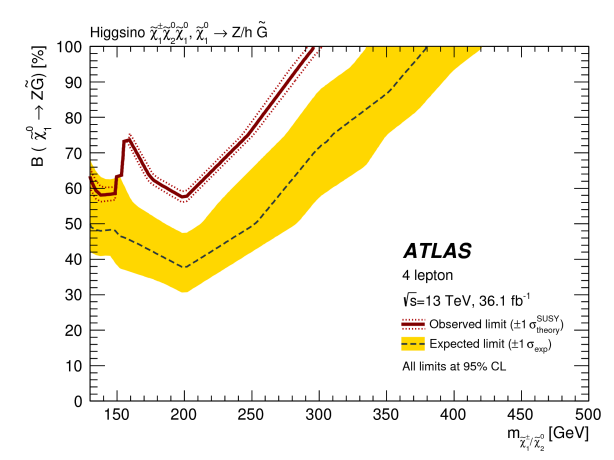

Figure 27: RPC GGM higgsino [21].

various signal scenarios by two kinds of SUSY productions (strong and electroweak processes), which remarkably extend the exclusion space of SUSY search. All these results are just based upon $1.2 \%$ of LHC planned total luminosity: $140 / 300 / 3000 \mathrm{fb}^{-1}$ at $\sqrt{s}=13-14 \mathrm{TeV}$ LHC data will come in the future years. So far SUSY is still an interesting scenario, with quite some parameter space left unconstrained by present searches, motivating further investigation.

\section{References}

[1] L. Evans and P. Bryant, LHC Machine, J. Inst. 3, S08001 (2008), doi:10.1088/17480221/3/08/S08001.

[2] [ATLAS Collaboration], The ATLAS Experiment at the CERN Large Hadron Collider, J. Inst. 3, S08003 (2008), doi:10.1088/1748-0221/3/08/S08003.

[3] [CMS Collaboration], The CMS experiment at the CERN LHC, J. Inst. 3, S08004 (2008), doi:10.1088/1748-0221/3/08/S08004.

[4] [ATLAS Collaboration], Search for squarks and gluinos in final states with hadronically decaying $\tau$-leptons, jets, and missing transverse momentum using $p p$ collisions at $\sqrt{s}=13 \mathrm{TeV}$ with the ATLAS detector, Phys. Rev. D 99, 012009 (2019), doi:10.1103/PhysRevD.99.012009.

[5] J. Alwall, M.-P. Le, M. Lisanti and J. G. Wacker, Searching for directly decaying gluinos at the Tevatron, Phys. Lett. B 666, 34 (2008), doi:10.1016/j.physletb.2008.06.065.

[6] J. Alwall, P. C. Schuster and N. Toro, Simplified models for a first characterization of new physics at the LHC, Phys. Rev. D 79, 075020 (2009), doi:10.1103/PhysRevD.79.075020.

[7] D. Alves et al., Simplified models for LHC new physics searches, J. Phys. G: Nucl. Part. Phys. 39, 105005 (2012), doi:10.1088/0954-3899/39/10/105005.

[8] M. Dine and W. Fischler, A phenomenological model of particle physics based on supersymmetry, Phys. Lett. B 110, 227 (1982), doi:10.1016/0370-2693(82)91241-2.

[9] L. Alvarez-Gaumé, M. Claudson and M. B. Wise, Low-energy supersymmetry, Nucl. Phys. B 207, 96 (1982), doi:10.1016/0550-3213(82)90138-9.

[10] C. R. Nappi and B. A. Ovrut, Supersymmetric extension of the $S U(3) \times S U(2) \times U(1)$ model, Phys. Lett. B 113, 175 (1982), doi:10.1016/0370-2693(82)90418-X. 
[11] [ATLAS Collaboration], Search for top squarks decaying to tau leptons in pp collisions at $\sqrt{s}=13 T e V$ with the ATLAS detector, Phys. Rev. D 98, 032008 (2018), doi:10.1103/PhysRevD.98.032008.

[12] M. Asano, H. Do Kim, R. Kitano and Y. Shimizu, Natural supersymmetry at the LHC, J. High Energ. Phys. 12, 019 (2010), doi:10.1007/JHEP12(2010)019.

[13] K. Inoue, A. Kakuto, H. Komatsu and S. Takeshita, Aspects of grand unified models with softly broken supersymmetry, Prog. Theor. Phys. 68, 927 (1982), doi:10.1143/PTP.68.927.

[14] J. Ellis and S. Rudaz, Search for supersymmetry in toponium decays, Phys. Lett. B 128, 248 (1983), doi:10.1016/0370-2693(83)90402-1.

[15] [CMS Collaboration], Search for supersymmetry in events with a $\tau$ lepton pair and missing transverse momentum in proton-proton collisions at $\sqrt{s}=13 \mathrm{TeV}$, J. High Energ. Phys. 11, 151 (2018), doi:10.1007/JHEP11(2018)151.

[16] M. Aaboud et al. [ATLAS Collaboration], Search for the direct production of charginos and neutralinos in final states with tau leptons in $\sqrt{s}=13 \mathrm{TeV} p p$ collisions with the ATLAS detector, Eur. Phys. J. C 78, 154 (2018), doi:10.1140/epjc/s10052-018-5583-9.

[17] A. Djouadi et al., The minimal supersymmetric Standard Model: Group summary report (1999), arXiv:hep-ph/9901246.

[18] C. F. Berger, J. S. Gainer, J. L. Hewett and T. G. Rizzo, Supersymmetry without prejudice, J. High Energ. Phys. 02, 023 (2009), doi:10.1088/1126-6708/2009/02/023.

[19] D. Alves et al., Simplified models for LHC new physics searches, J. Phys. G: Nucl. Part. Phys. 39, 105005 (2012), doi:10.1088/0954-3899/39/10/105005.

[20] [CMS Collaboration], Search for electroweak production of charginos and neutralinos in multilepton final states in proton-proton collisions at $\sqrt{s}=13 \mathrm{TeV}$, J. High Energ. Phys. 03, 166 (2018), doi:10.1007/JHEP03(2018)166.

[21] M. Aaboud et al. [ATLAS Collaboration], Search for supersymmetry in events with four or more leptons in $\sqrt{s}=13 \mathrm{TeV}$ pp collisions with ATLAS, Phys. Rev. D 98, 032009 (2018), doi:10.1103/PhysRevD.98.032009. 